\title{
Vector constants of the motion and orbits in the Coulomb/Kepler problem
}

\author{
Gerardo Muñoz \\ Department of Physics, California State University Fresno, Fresno, California 93740-0037
}

\section{Abstract}

The equation for the conic sections describing the possible orbits in a potential $V \sim r^{-1}$ is obtained by means of a vector constant of the motion differing from the traditional Laplace-RungeLenz vector. 
The Laplace-Runge-Lenz ${ }^{1,2,3,4}$ (or Hermann-Bernoulli-Laplace ${ }^{5}$ ) vector provides a simple and elegant way of obtaining the equation for the orbit of a particle moving under the influence of a radial inverse-square-law force. $\underline{6}$ This method also has the added advantage over the more common approach of direct integration of the equations of motion ${ }^{7}$ that the very existence of this single-valued constant of the motion explains the otherwise surprising degeneracy of the Coulomb/Kepler problem. $\stackrel{8}{-}$ Our aim here is to show that there is another vector constant of the motion which makes a first principles derivation of the equation of the orbit even easier than the Laplace-Runge-Lenz vector or the direct integration methods.

The force law $\mathbf{F}=-k \hat{\mathbf{r}} / r^{2}$ (we assume $k>0$; the repulsive case may be treated along the same lines) leads to the nonrelativistic equation of motion

$$
\frac{d \mathbf{p}}{d t}=-k \frac{\hat{\mathbf{r}}}{r^{2}}
$$

and to the well-known conservation laws for energy $E=\frac{1}{2} m v^{2}-k / r$ and angular momentum $\mathbf{L}=\mathbf{r} \times \mathbf{p}$. If we choose polar coordinates in the plane of the orbit, we have the usual expression $L=m r^{2} \dot{\theta}$, and also

$$
\frac{d \mathbf{p}}{d t}=m \frac{d \mathbf{v}}{d \theta} \dot{\theta}=\frac{d \mathbf{v}}{d \theta} \frac{L}{r^{2}} .
$$

Combining Eq. (2) with Eq. (1) we obtain

$$
\frac{d \mathbf{v}}{d \theta}=-\frac{k}{L} \hat{\mathbf{r}}
$$

Note that Eq. (3) implies immediately that $\mathbf{v}(0)=\mathbf{v}(2 \pi)$ and that the velocity vector traces out a circle if $\theta$ is allowed to vary from 0 to $2 \pi$. Integration of Eq. (3) is trivial if we recall that $d \hat{\boldsymbol{\theta}} / d \theta=-\hat{\mathbf{r}}$ :

$$
\mathbf{v}-\frac{k}{L} \hat{\boldsymbol{\theta}}=\mathbf{u},
$$

where $\mathbf{u}$ is a constant vector. This vector is the constant of the motion we propose to use instead of the Laplace-Runge-Lenz vector in the derivation of the orbits. It is interesting to note that a quaternion equivalent to Eq. (4) was already known to Hamilton in $1845.9 \frac{9}{}$ Unfortunately, this simple result seems to have vanished from textbooks on classical mechanics after the first decade of the twentieth century. ${ }^{10}$

The vector $\mathbf{u}$ may be evaluated in terms of the physical parameters of the problem. If we choose the angle so that the minimum value of $r$ is at $\theta=0$ and adjust our coordinate 
system so that $\mathbf{v}=v_{0} \hat{\mathbf{y}}$ at $r_{\min }$, we have

$$
\mathbf{u}=\left(v_{0}-\frac{k}{L}\right) \hat{\mathbf{y}}
$$

Because $L=m r_{\min } v_{0}$, the energy is $E=\frac{1}{2} m v_{0}^{2}-k / r_{\min }=\frac{1}{2} m v_{0}^{2}-k m v_{0} / L$. We solve this equation for $v_{0}$ and obtain

$$
v_{0}=\frac{k}{L} \pm \sqrt{\left(\frac{k}{L}\right)^{2}+\frac{2 E}{m}}=\frac{k}{L}(1 \pm \epsilon),
$$

where $\epsilon=\sqrt{1+2 E L^{2} / m k^{2}}$ will turn out to be the eccentricity. The plus sign is required at $r_{\text {min }}$; substituting this result into Eq. (5) provides us with an alternative form for $\mathbf{u}$ :

$$
\mathbf{u}=\frac{k}{L} \epsilon \hat{\mathbf{y}}
$$

Obtaining the equation of the orbit is now straightforward. By taking the scalar product of Eq. (4) with $\hat{\boldsymbol{\theta}}$ and using $\mathbf{v} \cdot \hat{\boldsymbol{\theta}}=r \dot{\theta}=L / m r, \hat{\mathbf{y}} \cdot \hat{\boldsymbol{\theta}}=\cos \theta$, we find

$$
\frac{L}{m r}-\frac{k}{L}=\frac{k}{L} \epsilon \cos \theta
$$

The definition $\alpha=L^{2} / m k$ allows us to write the solution for $r$ as

$$
r=\frac{\alpha}{1+\epsilon \cos \theta}
$$

which is the usual equation of a conic section with one focus at the origin and eccentricity $\epsilon$. Note that the velocity $\mathbf{v}=k[\epsilon \sin \theta \hat{\mathbf{r}}+(1+\epsilon \cos \theta) \hat{\boldsymbol{\theta}}] / L=k[-\sin \theta \hat{\mathbf{x}}+(\epsilon+\cos \theta) \hat{\boldsymbol{y}}] / L$ follows almost trivially from Eqs. (4) and (7). Hence the orbit in velocity space is always circular and characterized by $v_{x}^{2}+\left(v_{y}-k \epsilon / L\right)^{2}=k^{2} / L^{2}$. More precisely, the orbit in velocity space is a circle of radius $k / L$ and center at $(0, k \epsilon / L)$ if the orbit in position space is a circle $(\epsilon=0)$, an ellipse $(0<\epsilon<1)$, or a parabola $(\epsilon=1)$. On the other hand, if the spatial trajectory is a hyperbola $(\epsilon>1)$, the angle ranges from $-\cos ^{-1}(-1 / \epsilon)$ to $\cos ^{-1}(-1 / \epsilon)$ only, and the velocity space orbit is a section of a circle in the upper $v_{x}, v_{y}$ plane with $k\left(\epsilon^{2}-1\right) / \epsilon L<v_{y} \leq k(\epsilon+1) / L$.

The vector constant of the motion $\mathbf{u}$ is simpler (and its derivation certainly easier ${ }^{11}$ ) than the Laplace-Runge-Lenz vector, but the two are, of course, not independent constants of the motion. Indeed, the cross product of Eq. (4) with $m \mathbf{L}$ yields

$$
\mathbf{p} \times \mathbf{L}-m k \hat{\mathbf{r}}=m \mathbf{u} \times \mathbf{L} .
$$


The left-hand side is the Laplace-Runge-Lenz vector $\mathbf{A}$, so $\mathbf{A}=m \mathbf{u} \times \mathbf{L}$. The three conserved vectors $\mathbf{A}, \mathbf{u}$, and $\mathbf{L}$ form a right-handed orthogonal system equivalent to $\hat{\mathbf{x}}, \hat{\mathbf{y}}$, and $\hat{\mathbf{z}}$ after normalization.

As a final comment of pedagogical interest, we present a second approach that may be useful in an elementary discussion of the Coulomb/Kepler problem. We begin with the expression for the energy $E=\frac{1}{2} m v^{2}-k / r$. If we use $\mathbf{v} \cdot \hat{\boldsymbol{\theta}}=r \dot{\theta}=L / m r$ to replace $1 / r$ by $m \mathbf{v} \cdot \hat{\boldsymbol{\theta}} / L$ in the potential term, we may write $E$ as

$$
E=\frac{1}{2} m v^{2}-\frac{m k}{L} \mathbf{v} \cdot \hat{\boldsymbol{\theta}}
$$

If we complete the square in the velocity, we find

$$
E=\frac{1}{2} m u^{2}-\frac{m k^{2}}{2 L^{2}}
$$

with $\mathbf{u}$ the same vector as in Eq. (4). It follows that the magnitude of $\mathbf{u}$ must be constant. To prove that this vector is conserved both in magnitude and direction, we must only take a derivative of $\mathbf{u}$ and substitute $d \mathbf{v} / d t=-k \hat{\mathbf{r}} / m r^{2}$ and $d \hat{\boldsymbol{\theta}} / d t=-\dot{\theta} \hat{\mathbf{r}}$ into the result. The equation of the orbit may then be found as before. This approach does not require any integrations and amounts to a change of gauge from a scalar potential to an effective vector potential description of the problem.

1 P. S. Laplace, Celestial Mechanics (Chelsea, NY, 1969), Vol. 1, p. 344, Eqs. [572].

2 C. Runge, Vektoranalysis (Hirzel, Leipzig, 1919), Vol. 1, p. 70.

3 W. Lenz, "On the Course of the Motion and the Quantum States of the Disturbed Kepler Motion," Z. Phys. 24, 197-207 (1924).

4 H. Goldstein, Classical Mechanics (Addison-Wesley, Reading, MA, 1980), 2nd ed.

5 H. Goldstein, "More on the prehistory of the Laplace or Runge-Lenz vector," Am. J. Phys. 44, 1123-1124 (1976).

6 For a three-dimensional Hamiltonian system with $H=p^{2} / 2 m+V(r, \theta, \phi)$, the only potential possessing a Laplace-Runge-Lenz-type constant of the motion is $V(r, \theta, \phi)=-k / r$. See V. M. Gorringe and P. G. L. Leach, "The first integrals and their Lie algebra of the most general autonomous Hamiltonian of the form $H=T+V$ possessing a Laplace-Runge-Lenz vector," J. Austral. Math. Soc. Ser. B 34, 511-522 (1993). 
7 See, for example, J. B. Marion and S. T. Thornton, Classical Dynamics of Particles and Systems (Saunders College Publishing, 1995), 4th ed; K. R. Symon, Mechanics (Benjamin Cummings, 1971), 3rd ed. The method presented in these textbooks is due to Johann Bernoulli. For an interesting historical account, see E. J. Aiton, "The contributions of Isaac Newton, Johann Bernoulli and Jakob Hermann to the inverse problem of central forces," Studia Leibnitiana, Sonderheft 17, 48-58, edited by H.-J. Hess and F. Nagel (Franz Steiner Verlag Wiesbaden GMBH, Stuttgart 1989).

8 Vector constants of the motion are known to exist for any potential that conserves at least the direction of the angular momentum. See D. M. Fradkin, "Existence of the dynamic symmetries $\mathrm{O}_{4}$ and $\mathrm{SU}_{3}$ for all classical potential problems," Prog. Theor. Phys. 37, 798-812 (1967); T. Yoshida, "Determination of the generalized Laplace-Runge-Lenz vector by an inverse matrix method," Am. J. Phys. 57, 376-377 (1989); C. C. Yan, "Determination of vector constant of motion for a particle moving in a conservative force field," J. Phys. A: Math. Gen. 24, 47314738 (1991). However, these constants of the motion do not entail a degeneracy unless they are single-valued or at most finitely-multivalued. See P. Stehle and M. Y. Han, "Symmetry and degeneracy in classical mechanics," Phys. Rev. 159, 1076-1082 (1967).

9 W. R. Hamilton, "On the applications of the method of quaternions to some dynamical questions," in The Mathematical Papers of Sir William Rowan Hamilton, edited by H. Halberstam and R. E. Ingram (Cambridge University Press, 1967), Vol. III, pp. 441-448.

10 A number of alternative formulations of Eq. (4) have appeared in the pages of this journal, however. See H. Abelson, A. diSessa, and L. Rudolph, "Velocity space and the geometry of planetary orbits," Am. J. Phys. 43, 579-589 (1975); R. P. Patera, "Momentum-space derivation of the Runge-Lenz vector," Am. J. Phys. 49, 593-594 (1981); D. Derbes "Reinventing the wheel: Hodographic solutions to the Kepler problems," Am. J. Phys. 69, 481-489 (2001). The present note should be regarded as a brief complement to these interesting papers.

11 A derivation of the Laplace-Runge-Lenz vector from first principles may be found in H. Kaplan, "The Runge-Lenz vector as an "extra" constant of the motion," Am. J. Phys. 54, 157-161 (1986). The method Kaplan attributes to E. T. Whittaker was in effect used by Laplace in Ref. 1 over a century earlier. 